\title{
Avaliação da retinopatia hipertensiva através do potencial oscilatório do eletrorretinograma
}

\author{
Evaluation of hypertensive retinopathy through the oscillatory potentials \\ of the electroretinogram
}

\author{
Alan Diego Neg'retto ${ }^{1}$ \\ Alexandre Antônio Marques Rosa ${ }^{2}$ \\ Augusto Akio Nakashima ${ }^{3}$ \\ Kátia C.Orteg'a ${ }^{4}$ \\ Décio Mion Júnior ${ }^{5}$ \\ Maria Kiyoko Oyamada ${ }^{6}$ \\ Yoshitaka Nakashima ${ }^{7}$
}

Trabalho realizado no Hospital das Clínicas da Faculdade de Medicina da Universidade de São Paulo - USP - São Paulo (SP) - Brasil.

${ }^{1}$ Pós-graduando da Faculdade de Medicina da Universidade de São Paulo - USP - São Paulo (SP) - Brasil.

${ }^{2}$ Doutor, Departamento de Oftalmologia do Hospital Universitário Bettina Ferro de Sousa da Universidade Federal do Pará - UFPA - Belém (PA) - Brasil.

${ }^{3}$ Acadêmico da USP - São Paulo (SP) - Brasil.

${ }^{4}$ Pós-graduanda da USP - São Paulo (SP) - Brasil.

${ }^{5}$ Livre docente, Professor da Disciplina de Nefrologia da USP - São Paulo (SP) - Brasil.

${ }^{6}$ Doutora, Médica Assistente da Clínica Oftalmológica do Hospital das Clínicas da USP - São Paulo (SP) Brasil.

${ }^{7}$ Doutor, Médico Assistente da Clínica Oftalmológica do Hospital das Clínicas da USP - São Paulo (SP) Brasil.

Endereço para correspondência: Alan Diego Negretto. Rua Dona Avelina, 77/161 - São Paulo (SP)

CEP 04111-010

E-mail: alan_negretto@terra.com.br

Recebido para publicação em 16.03.2006

Última versão recebida em 04.07.2007

Aprovação em 17.07.2007

Nota Editorial: Depois de concluída a análise do artigo sob sigilo editorial e com a anuência do Dr. André Messias sobre a divulgação de seu nome como revisor, agradecemos sua participação neste processo.

\section{RESUMO}

Objetivo: Avaliar o comportamento do potencial oscilatório escotópico do eletrorretinograma de campo total (ERG) na retinopatia hipertensiva. Métodos: Quarenta e quatro pacientes foram submetidos à avaliação clínica e subdivididos em dois grupos: 26 hipertensos (HT) com média de idade de $52,23 \pm 5,79$ anos divididos em 10 homens $(38,46 \%)$ e 16 mulheres $(61,54 \%)$ e 18 normotensos (NT) com média de idade de 51,79 $\pm 10,23$ anos divididos em 5 homens $(27,78 \%)$ e 13 mulheres $(72,22 \%)$. Foram incluídos no estudo apenas hipertensos leves a moderados (estágio 1 e 2 respectivamente) sem lesões em outro órgão-alvo.Os pacientes hipertensos foram mantidos sob placebo durante o período do estudo. Em seguida, foram submetidos à avaliação oftalmológica e realização do ERG. O eletrorretinograma de campo total (ERG), com registro das respostas: escotópica, escotópica máxima, PO escotópico, fotópica e "flicker". Para análise da resposta do PO foi considerada a latência dos dois primeiros picos e o valor médio da amplitude dos três picos do complexo de três respostas consecutivas, denominado índice oscilatório (IO). Resultados: A hipertensão arterial acometia $26(59,1 \%)$ dos pacientes, ao passo que $18(40,9 \%)$ eram normotensos. A média do IO obtido foi de $257,41 \mu \mathrm{V}$ no grupo de NT e de $217,81 \mu \mathrm{V}$ no HT ( $\mathrm{p}=0,006)$. As médias de latências obtidas para os picos 1 (NT-18,42 ms e HT-17,91 ms) e 2(NT-24,54 ms e HT- 24,29 ms) não foram diferentes entre os grupos ( $p>0,05)$. Conclusão: Os hipertensos apresentam índice oscilatório significativamente menor que os normotensos, sugerindo que a hipertensão arterial pode ocasionar disfunção da retina interna.

Descritores: Hipertensão/complicações; Eletrorretinografia; Doenças retinianas

\section{INTRODUÇÃO}

A hipertensão arterial acomete cerca de $30 \%$ da população brasileira adulta, sendo considerada como um dos principais fatores de morbidade e mortalidade cardiovasculares ${ }^{(1)}$.

O exame de fundo do olho constitui um importante parâmetro para avaliação das doenças vasculares sistêmicas, sendo a única região do corpo onde o médico pode visualizar diretamente as alterações micro e macrovasculares. Como as anormalidades dos vasos retinianos são relativamente inespecíficas, seu significado deve ser avaliado dentro do contexto de outros sintomas e sinais sistêmicos e oculares ${ }^{(2-3)}$.

No protocolo básico ou standard do eletrorretinograma de campo total (ISCEV), cinco tipos diferentes de resposta são obtidos, conforme o estí- 
mulo utilizado e o estado de adaptação do olho: 1) resposta dos bastonetes/adaptado ao escuro (estímulo branco fraco); 2) resposta de cones e bastonetes/adaptado ao escuro (estímulo branco intenso); 3) potenciais oscilatórios (POs)/adaptado ao escuro (estímulo branco intenso); 4) resposta dos cones/adaptado à luz (estímulo branco único); 5) resposta dos cones ao "flicker"/adaptado à luz (seqüência de estímulos na freqüência de $30 \mathrm{~Hz})^{(4)}$.

A origem dos potenciais oscilatórios não está muito bem definida, mas parecem ser gerados nas camadas retinianas internas, células amácrinas e interplexiformes, que são supridas pelo sistema vascular retiniano ${ }^{(5-6)}$. Sua amplitude está reduzida em todas as formas graves de angiopatia.

Estudo realizado em 2001, comparou o olho contralateral de 59 pacientes que tiveram oclusões vasculares e encontrou respostas dos POs significativamente inferiores nos olhos acometidos, principalmente nos casos de oclusão da veia central da retina $(\mathrm{OVCR})^{(7)}$.

Além disso, os POs podem estar reduzidos em formas menos graves de angiopatia retiniana, como nos estágios iniciais da retinopatia diabética sem alterações fundoscópicas evidentes, sendo considerados marcadores das alterações da microcirculação e da progressão da doença ${ }^{(8-9)}$.

O objetivo desta análise é descrever alterações nos potenciais oscilatórios em pacientes com hipertensão arterial leve a moderada, na ausência de sinais de retinopatia hipertensiva, como marcador precoce do acometimento da circulação retiniana.

\section{MÉTODOS}

\section{Pacientes}

Foram incluídos indivíduos de ambos os sexos; sem restrição de raça; maiores que 30 anos, com índice de massa corpórea abaixo de $30 \mathrm{~kg} / \mathrm{m}^{2}$. Foram excluídos pacientes com opacidades de meios que impedissem a visualização da retina (catarata avançada, hemorragia vítrea e leucoma), doenças retinianas que sabidamente alteram o ERG de campo total (distrofias retinianas), uveíte posterior, glaucoma, alta miopia, uso de drogas tóxicas para a retina (cloroquina, etc); resposta anormal dos outros componentes do ERG; gravidez e lactação; obesidade; diabete melito (teste de tolerância à glicose anormal ou duas glicemias de jejum > $126 \mathrm{mg} / \mathrm{dL}$, ou uso de hipoglicemiante oral ou insulina).

O estudo foi transversal, observacional do tipo caso-controle. Os pacientes foram avaliados na Unidade de Hipertensão Arterial do Hospital das Clínicas, Disciplina de Nefrologia da Faculdade de Medicina da Universidade de São Paulo para avaliação clínica e diagnóstico de hipertensão arterial.

A pressão arterial foi medida segundo os critérios estabelecidos pela IV Diretrizes Brasileiras de Hipertensão Arterial ${ }^{(1)}$.

Hipertensão arterial foi definida como pressão sistólica maior ou igual a $140 \mathrm{mmHg}$ e/ou pressão diastólica maior ou igual a $90 \mathrm{mmHg}$, e normotensão quando pressão sistó- lica/diastólica menor de 140/90 mmHg, sem uso de medicamento.

Foram incluídos no estudo apenas pacientes hipertensos essenciais (não secundários) sem sinais de retinopatia hipertensiva ou lesão em outro órgão-alvo. O estadiamento seguiu a classificação Brasileira ${ }^{(1)}$ onde incluíram-se:

Hipertensos estágio 1 (leves): pressão sistólica (140-159 $\mathrm{mmHg}$ ) e pressão diastólica $(90-99 \mathrm{mmHg})$.

Hipertensos estágio 2 (moderados): pressão sistólica (160-179 mmHg) e pressão diastólica (100-109 mmHg). Sem lesões em outro órgão-alvo.

Quarenta e quatro pacientes $(n=44)$ foram submetidos à avaliação clínica e subdivididos em 2 grupos: 26 hipertensos (HT) com média de idade de 52,23 $\pm 5,79$ anos divididos em 10 homens $(38,46 \%)$ e 16 mulheres $(61,54 \%)$ e 18 normotensos (NT)) com média de idade de 51,79 \pm 10,23 anos divididos em 5 homens $(27,78 \%)$ e 13 mulheres $(72,22 \%)$.

Também foram definidos como hipertensos, pacientes normotensos em uso de medicação anti-hipertensiva. Com a permissão da Comissão de Ética do Hospital das Clínicas da FMUSP os pacientes hipertensos receberam placebo durante 30 dias para evitar possível influência da medicação sobre o registro do ERG.

Em seguida, os pacientes foram encaminhados para avaliação oftalmológica Clínica Oftalmológica do Hospital das Clínicas da Faculdade de Medicina da USP, que consistiu de medida da acuidade visual com tabela de Snellen, refratometria, biomicroscopia de segmento anterior e de fundo, tonometria de aplanação e oftalmoscopia binocular indireta.

\section{ERG}

Após dilatação pupilar ampla ( $>8 \mathrm{~mm}$ ) obtida com colírio de tropicamida $1 \%$ e fenilefrina $10 \%$, eletrodos foram posicionados, no lobo da orelha direita (terra) e na fronte, a cerca de $3 \mathrm{~cm}$ do násion (referência). Eletrodos corneanos do tipo ERG-jet (ativo), foram fixados centralmente na córnea com auxílio de colírio de metilcelulose a $2 \%$, em superfície previamente anestesiada com colírio de proparacaína a $0,5 \%$.

Respeitando-se os tempos de adaptação ao claro e ao escuro, realizado o ERG nas seguintes etapas: escotópico, resposta máxima combinada, potenciais oscilatórios, fotópico e "flicker". Para cada fase foram colhidas quatro respostas, com intervalo de 15 segundos, e considerado para análise a média dos valores de latência e amplitude obtidos.

$\mathrm{O}$ protocolo seguiu as recomendações do padrão da International Society of Clinical Electrophysiology of Vision (ISCEV), adaptação ao escuro por 20 minutos (ERG escotópico) e em seguida, adaptação ao claro por 10 minutos com luminância de $24,8 \mathrm{~cd} / \mathrm{m}^{2}$. Os exames foram realizados pela segunda autora (MKO), utilizando-se o equipamento Epic 2000 - LKC, com cúpula de Ganzfield semi-automática. Para o PO escotópico foram utilizados filtros de passa-faixa entre $75 \mathrm{~Hz}$ (nível inferior) e $500 \mathrm{~Hz}$ (nível superior) e estímulo de luz branca de $2.387 \mathrm{~cd} / \mathrm{m}^{2}$, com duração de $3,5 \mathrm{~ms}$. 
Para análise da resposta do PO foram considerados o índice oscilatório (IO) e a latência dos dois primeiros picos positivos (Figura 1 - P1 E P2). Para facilitar a análise, convencionou-se que as respostas seriam obtidas a partir do olho direito (OD) de cada paciente. O valor obtido pelo cálculo da média da amplitude, dos três primeiros picos do complexo, de três respostas consecutivas (descartando-se a primeira das quatro respostas obtidas), foi denominado nesta pesquisa de IO (Figura 1).

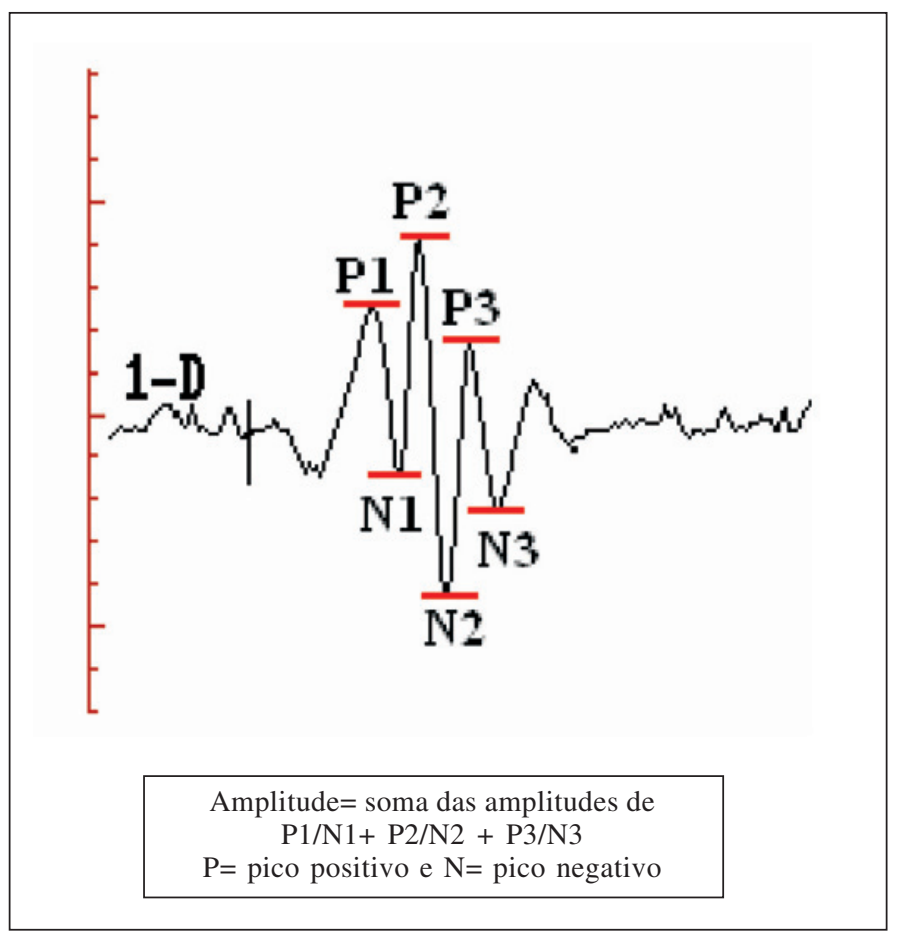

Figura 1 - Realização do cálculo do 10 a partir de exemplos de respostas obtidas entre pacientes de cada grupo estudado
As médias do IO e latências de P1 e P2 dos potenciais oscilatórios foram comparadas por meio do teste $t$ de Student e o nível de significância foi $\mathrm{p}<0,05$.

O protocolo de pesquisa foi aprovado pela Comissão de Ética do Hospital das Clínicas da Faculdade de Medicina da Universidade de São Paulo. Todos os indivíduos assinaram o termo de consentimento livre e esclarecido após explicação e concordância da participação no estudo.

\section{RESULTADOS}

A hipertensão arterial acometia $26(57,8 \%)$ dos pacientes, enquanto $19(42,2 \%)$ eram normotensos.

Todos os hipertensos tinham o diagnóstico há mais de um ano.

No comparativo entre os grupos, a média de idade dos pacientes foi de 52,23 \pm 8,95 anos (HT) e 50,56 \pm 5,79 anos $(\mathrm{NT}) ;(\mathrm{p}=0,885)$.

A acuidade visual corrigida foi de $20 / 20(1,0)$ em todos os pacientes estudados (100\%).

$\mathrm{Na}$ oftalmoscopia binocular indireta não foram constatados sinais de retinopatia hipertensiva em nenhum dos pacientes avaliados pelo mesmo examinador (AAMR).

Houve diferença nas respostas do IO entre o grupo de hipertensos (Figura 2A) e o grupo de normotensos (Figura 2B).

A diferença das médias do IO foi estatisticamente significativa entre os grupos estudados (Tabela 1).

A latência média obtida para o pico P1 e P2 foram respectivamente de $17,91 \mathrm{~ms}$ e de $24,29 \mathrm{~ms}$ no grupo de HT e 18,42 ms e de 24,54 ms no grupo de NT, com $\mathrm{p}=0,154$ e $\mathrm{p}=0,598$.

Não foram observadas diferenças nas amplitudes ou latências das respostas do ERG escotópico: resposta dos bastonetes ou resposta máxima combinada, nem no ERG fotópico: flash único ou "flicker" entre os grupos estudados.

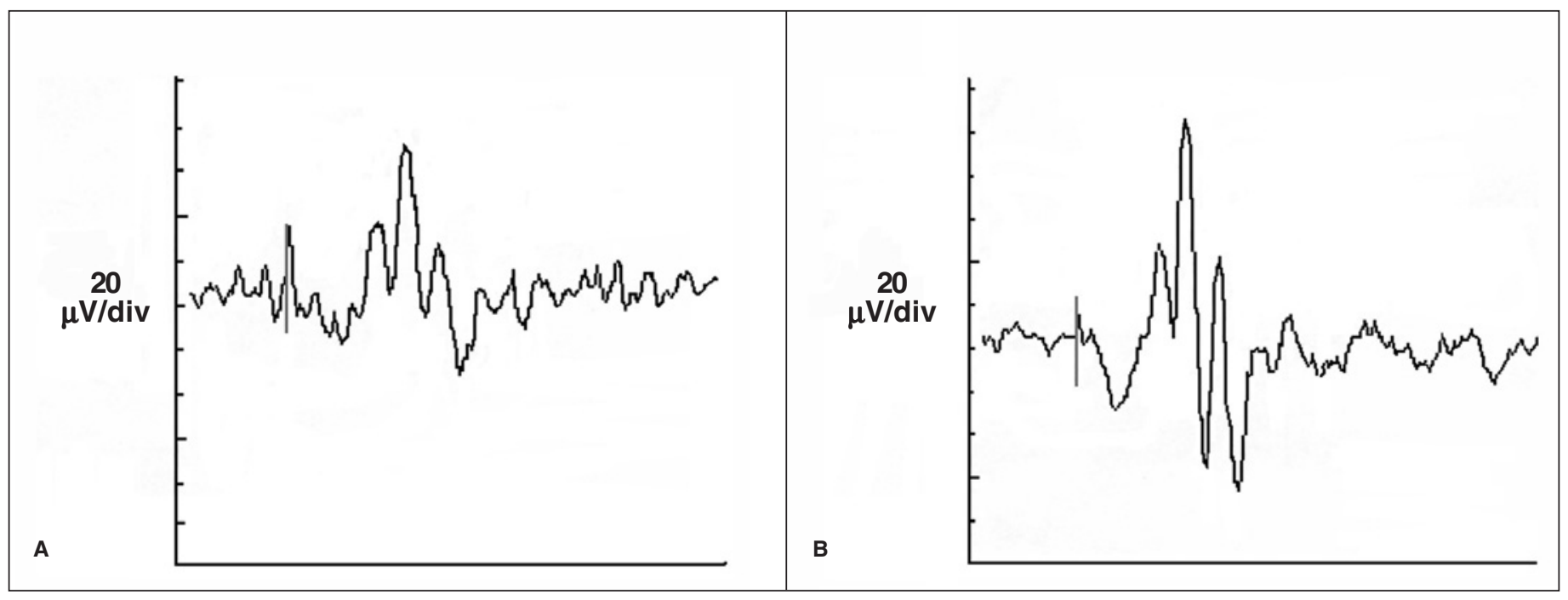

Figura 2 - A) Hipertensos; B) Normotensos 


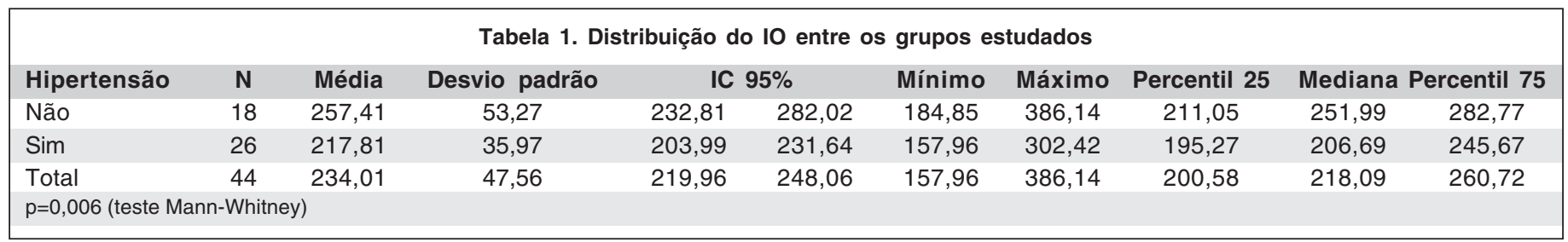

\section{DISCUSSÃO}

O principal achado deste estudo foi que os hipertensos apresentaram índice de amplitude dos potenciais oscilatórios do ERG significativamente menor que os normotensos.

Embora a origem exata dos POs do ERG seja ainda motivo de discussão, sua correlação com distúrbios da circulação retiniana e camadas internas têm sido claramente demonstrada ${ }^{(10-11)}$. Sua amplitude é reduzida em todas as formas de angiopatia, como nas oclusões arteriais ou venosas, doença de Takayasu e diabetes, refletindo distúrbios na função das camadas internas da retina que podem ter sido causadas por insuficiência na circulação retiniana e/ou comprometimentos graves da microcirculação retiniana que promovem uma redução seletiva do PO do ERG de campo total, mantendo-se a integridade da resposta dos fotorreceptores, com ondas a e $\underline{b}$ de latência e amplitude dentro da normalidade ${ }^{(11)}$.

Em 1984, estudos distintos, concluíram que nos hipertensos arteriais a alteração do PO, caracterizado pela redução da amplitude, ocorre muito antes dos mesmos apresentarem alterações retinianas detectáveis à fundoscopia ${ }^{(12-13)}$.

Nosso estudo confirma outros achados ${ }^{(14)}$ que demonstraram haver redução precoce do IO nos indivíduos portadores de hipertensão essencial. Esta alteração precede as alterações morfológicas peculiares da retinopatia hipertensiva e são sugestivas de comprometimento precoce na dinâmica da circulação microvascular nos hipertensos.

Aumento de latência associado à redução da amplitude tem sido descrito nas formas graves de angiopatias. No presente estudo foram avaliados somente pacientes com hipertensão leve a moderada e não observamos aumento de latência dos picos P1 e P2 nos hipertensos.

Considerando os achados ora obtidos, assim como os observados na literatura, importância deve ser dada aos subcomponentes do ERG de campo total, quer do ponto de vista de avaliação diagnóstica quanto para o seguimento clínico nas doenças vasculares crônicas retinianas. Talvez a alteração do IO possa constituir um dado de importância na avaliação de lesão em órgão-alvo. Mas para se obter uma correlação dos achados quanto ao comprometimento do PO na retinopatia hipertensiva seria necessário estudo com maior casuística, considerando-se o estadiamento da retinopatia e a severidade da doença hipertensiva arterial sistêmica.

\section{CONCLUSÃO}

Os hipertensos apresentam índice de amplitude dos potenciais oscilatórios do ERG significativamente menor que os normotensos, sugerindo que a hipertensão arterial pode ocasionar disfunção das camadas internas da retina que pode ter sido causada por insuficiência da sua microcirculação.

\section{ABSTRACT}

Purpose: To evaluate the behavior of the scotopic Oscillatory Potentials (OP) of total field electroretinogram (ERG) in hypertensive retinopathy. Methods: Forty-four patients $(n=44)$ were submitted to clinical evaluation and subdivided in to 2 groups: hypertensives (HT) and normotensives (NT). The hypertensives patients were maintained under placebo during the period of the study. Soon afterwards, they were submitted to ophthalmological evaluation and accomplishment of ERG. Total field electroretinogram (ERG), with recording of the answers: scotopic, maximum scotopic, OP scotopic, photopic and flicker. For analysis of the $\mathrm{OP}$ answer the latency of the first 2 peaks and the average value of the width of the first 3 peaks of the compound of 3 consecutive answers, denominated Oscillatory Index (OI) were considered. Results: The sample was composed of 44 patients, with a mean age of $51.55 \pm 7.2$ range (34 to 68) years, 24 being females. Arterial hypertension affected $26(59.1 \%)$ of the patients, while $18(40.9 \%)$ were normotensives. The average of the obtained IO was $257.41 \mu \mathrm{v}$ in the NT group and of $217.81 \mu \mathrm{v}$ in HT ( $=0.006)$. The averages of latencies obtained for peaks 1 (NT-18.42 and HT-17.91) and 2 (NT-24.54 and HT - 24.29) were not different between the groups $(\mathrm{p}>0.05)$. Conclusions: The hypertensive patients presented significantly smaller oscillatory index than the normotensives, suggesting that arterial hypertension might cause dysfunction of the internal retina.

Keywords: Hypertension/complications; Electroretinography; Retinal diseases

\section{REFERÊNCIAS}

1. Sociedade Brasileira de Hipertensão; Sociedade Brasileira de Cardiologia; Sociedade Brasileira de Nefrologia. IV Diretrizes Brasileiras de Hipertensão Arterial. Arq Bras Cardiol. 2004;82(Supl 4):7-22. 
2. Ashton N. The eye in malignant hypertension. Trans Am Acad Ophthalmol Otolaryngol. 1972;76(1):17-40.

3. Hayreh SS, Servais GE, Virdi PS. Retinal arteriolar changes in malignant arterial hypertension. Ophthalmologica. 1989;198(4):178-96.

4. Standard for clinical electroretinography. International Standardization Committee. Arch Ophthalmol. 1989;107(6):816-9.

5. Cobb WA, Morton HB. A new component of the human electroretinogram. J Physiol. 1953;123:36-7.

6. Miyake Y. Macular oscillatory potentials in humans. Macular Ops. Doc Ophthalmol. 1990;75(2):111-24.

7. Huang S, Wu L, Luo T, Wu DZ, Jiang F, Luo G, Ma J. The electroretinogram in patients with retinal vascular occlusion. Yan Ke Xue Bao. 2001;17(1):50-3.

8. Speros P, Price J. Oscillatory potentials. History, techniques and potential use in the evaluation of disturbances of retinal circulation. Surv Ophthalmol. 1981;25(4):237-52.

9. Bresnick GH, Korth K, Groo A, Palta M. Electroretinographic oscillatory potentials predict progression of diabetic retinopathy. Preliminary report. Arch Ophthalmol. 1984;102(9):1307-11.

10. Nobre F. Análise dos dados obtidos e emissão de laudos. In: Mion Júnior D, Nobre F, Oigman W. MAPA: Monitorização ambulatorial da pressão arterial. $2^{\underline{a}}$ ed. São Paulo: Atheneu; 1998. p.211-24.

11. de Venecia G, Wallow I, Houser D, Wahlstrom M. The eye in accelerated hypertension. I. Elschnig's spots in nonhuman primates. Arch Ophthalmol. 1980;98(5):913-8.

12. Bellini G, Bocin E, Cosenzi A, Sacerdote A, Molino R, Solimano N, Ravalico G. Oscillatory potentials of the electroretinogram in hypertensive patients. Hypertension. 1995;25(4 Pt 2):839-41.

13. Ravalico G, Rinaldi G, Solimano N, Bellini G, Cosenzi A, Sacerdote A, Bocin E. Oscillatory potentials in subjects with treated hypertension. Ophthalmologica. 1995;209(4):187-9.

14. Bellini G, Bocin E, Cosenzi A, Sacerdote A, Molino R, Solimano N, Ravalico G. Oscillatory potentials of the electroretinogram in hypertensive patients. Hypertension. 1995;25(4 Pt 2):839-41.

\title{
Congresso Sotrim Sociedade de Oftalmologia do Triângulo Mineiro
}

\section{3 a 25 de outubro de 2008 Center Convention Uberlândia - MG}

\author{
INFORMAÇÕES \\ Sion Eventos \\ Tel.: (34) 3231-4500 \\ e-mail: fernando@sioneventos.com.br
}

